\title{
ESTIMATES FOR THE POISSON KERNELS AND A FATOU TYPE THEOREM APPLICATIONS TO ANALYSIS ON SIEGEL DOMAINS
}

\author{
ANDRZEJ HULANICKI \\ Instytut Matematyczny, Uniwersytet Wroctawski \\ Pl. Grunwaldzki 2/4, 50-384 Wroctaw, Poland \\ E-mail: hulanick@math.uni.wroc.pl
}

Abstract. This is a short description of some results obtained by Ewa Damek, Andrzej Hulanicki, Richard Penney and Jacek Zienkiewicz. They belong to harmonic analysis on a class of solvable Lie groups called $N A$. We apply our results to analysis on classical Siegel domains.

1. $N A$ groups. Let $\mathbf{s}$ be a solvable Lie algebra. We assume that $\mathbf{s}$ as a linear space is the direct sum of two subalgebras

$$
\mathbf{s}=\mathbf{n} \oplus \mathbf{a},
$$

where $\mathbf{n}$ is nilpotent and a Abelian. We assume that there exists a basis $E_{1}, \ldots, E_{n}$ of $\mathbf{n}$ such that for every $H$ in $\mathbf{a}$

$$
\left[H, E_{j}\right]=\left\langle\lambda_{j}, H\right\rangle E_{j}, \quad \lambda_{j} \in \mathbf{a}^{*}, j=1, \ldots, n .
$$

We call $\lambda_{j}$ 's the roots. For $\lambda \in\left\{\lambda_{1}, \ldots \lambda_{n}\right\}=\Lambda$ let

$$
\mathbf{n}^{\lambda}=\left\{Y \in \mathbf{n}: \operatorname{ad}_{H} Y=\langle\lambda, H\rangle Y\right\} \text { for all } H \in \mathbf{a} .
$$

We say that a subspace $\mathbf{n}^{\prime}$ of $\mathbf{n}$ is homogeneous, if for every $H$ in $\mathbf{a}$

$$
\operatorname{ad}_{H} \mathbf{n}^{\prime} \subset \mathbf{n}^{\prime} .
$$

Let

$$
S=\exp \mathbf{s}, N=\exp \mathbf{n} \text { and } A=\exp \mathbf{a} \text {. }
$$

1991 Mathematics Subject Classification: Primary: 32A07, 32M10. Secondary: 22E25.

Extended version of the lecture given at the Banach Center Colloquium on 18th November 1993.

Supported by KBN grant No. 2 P 30105107.

The paper is in final form and no version of it will be published elsewhere. 
Then

$$
S=N A
$$

is a semidirect product of the groups $N$ and $A, A$ acting on $N$ by

$$
a \exp \left\{\sum x_{j} E_{j}\right\} a^{-1}=\exp \left\{\sum x_{j} e^{\left\langle\lambda_{j}, \log a\right\rangle} E_{j}\right\} .
$$

In general, $A$ is multidimensional, hence for $a \in A \log a$ is a vector. We say that $a \rightarrow 0$ with respect to a subset $\Lambda_{1}$ of $\Lambda$, if $\langle\lambda, \log a\rangle \rightarrow-\infty$ for $\lambda \in \Lambda_{1}$. Then, of course,

if $a \rightarrow 0$ with respect to $\Lambda_{1}$.

$$
a \exp \left\{\sum x_{j} E_{j}\right\} a^{-1} \rightarrow e,
$$

The name $N A$ comes from the main source of examples of such groups: the $N A$ part of the Iwasawa decomposition of a semisimple (non-compact, finite center) group: $N A K$. We note that the symmetric space $N A K / K$ admits a simply transitive group of isometries of the form $N A$ acting on the left.

But also every proper homogeneous cone $\Omega$ in $\mathbf{R}^{n}$ admits a simply transitive group of linear transformations which is of the form $N A,[\mathrm{~V}]$.

Every bounded homogeneous domain $D \subset \mathbf{C}^{n}$ admits a simply transitive group of biholomorphic transformations of the form $N A,[\mathrm{~V}]$.

All known examples of non-compact Riemannian harmonic spaces, also the non-symmetric ones produced by E. Damek and F. Ricci, have the form $N A, N$ being a so called group of the Heisenberg type, [DR1], [DR2].

Let us look at the following four examples:

EXAMPLE 1. Let

$$
D=\{z \in \mathbf{C}: \Im z>0\} .
$$

We consider the following two groups of biholomorphic maps of $D$ onto itself:

$$
N=\mathbf{R} \text { and } A=\mathbf{R}^{+} \text {. }
$$

$N$ acts on $D$ by translations parallel to the real axis:

$$
x_{0} \cdot z=x_{0}+z,
$$

$A$ acts by dilations:

$$
a \cdot z=a z \text {. }
$$

The group generated by these two groups of transformations is denoted by $N A$. Of course

$$
N A=N \times A \text { as a manifold }
$$

and the group multiplication is

$$
x a \cdot y b=(x+a y) a b .
$$

We also see that the action of $N A$ on $D$ is simply transitive:

$$
x a \cdot i=x+i a .
$$


We note that our group $N A$ acts also on the boundary $B=\{z: \Im z=0\}$ of $D$ by affine transformations.

EXAMPLE 2. Let

$$
D=\left\{\left(w^{\prime}, w\right) \in \mathbf{C} \times \mathbf{C}^{n}: \Im w^{\prime}-\sum\left|w_{j}\right|^{2}>0\right\} .
$$

As the upper half-plane is biholomorphic with the unit disc, the corresponding Cayley transformation maps the unit ball $B \subset \mathbf{C} \times \mathbf{C}^{n}$ biholomorphically onto $D$ :

$$
\mathbf{C} \times \mathbf{C}^{n} \ni\left(w^{\prime}, w\right) \rightarrow\left(\frac{w^{\prime}+i}{1+i w^{\prime}}, \frac{w}{1+i w^{\prime}}\right) \in \mathbf{C} \times \mathbf{C}^{n} .
$$

Again there is a group $N$ of transformations acting on $D$ "parallel to the boundary"

$$
B=\left\{\left(w^{\prime}, w\right) \in \mathbf{C} \times \mathbf{C}^{n}: \Im w^{\prime}-\sum\left|w_{j}\right|^{2}=0\right\} .
$$

Here $N$ is $\mathbf{H}^{n}$ the Heisenberg group. As a manifold $N=\mathbf{R} \times \mathbf{C}^{n}$, the multiplication is defined by $(x, z)(y, w)=\left(x+y+2 \Im \sum w_{j}^{\prime} \bar{w}_{j}, w^{\prime}+w\right)$. The action of $N$ on $D$ is given by the formula

$$
N \times D \ni\left((x, u),\left(w^{\prime}, w\right)\right) \rightarrow\left(w^{\prime}+x+2 i \Phi(w, u)+i \Phi(u, u), w+u\right) \in D,
$$

where $\Phi(u, w)=\sum u_{j} \bar{w}_{j}$. A simple calculation shows that in fact the action of $N$ on $\mathbf{C}^{n+1}$ preserves the form

$$
\rho\left(w^{\prime}, w\right)=\Im w^{\prime}-\sum\left|w_{j}\right|^{2},
$$

thus it maps $D$ onto $D$ and also $B$ onto $B$.

The group $A$ is again equal to $\mathbf{R}^{+}$. $A$ acts on $D$ by non-isotropic dilations:

$$
a \cdot\left(w^{\prime}, w\right) \rightarrow\left(a w^{\prime}, a^{\frac{1}{2}} w\right) .
$$

Under this action the form $\rho$ transforms as follows:

$$
\rho\left(a w^{\prime}, a^{\frac{1}{2}} w\right)=a \rho\left(w^{\prime}, w\right),
$$

hence $A$ also preserves both $D$ and $B$.

We see that the group $N A$ of transformations of $D$ generated by $N$ and $A$ is the group which contains $N$ as a normal subgroup of codimension 1 and the group $A$ acts as a group of automorphisms of $N$ by non-isotropic dilations. Moreover, the group $N A$ acts simply transitively on the domain $D$ as a group of biholomorphic transformations.

EXAMPLE 3. Let $M$ be the space of symmetric real $n \times n$ matrices and let $\Omega$ be the open cone in $M$ consisting of positive definite matrices. Let

$$
D=M+i \Omega \subset \mathbf{C}^{d}, d=\frac{(n+1) n}{2} .
$$

To relate a $N A$ group to this example we proceed as follows. Let $E_{i j}$ be an elementary $n \times n$ matrix. We put

$$
\mathbf{a}=\operatorname{lin}\left\{E_{11}, \ldots, E_{n n}\right\}
$$




$$
\begin{gathered}
\mathbf{n}_{0}=\operatorname{lin}\left\{E_{i j}: i<j\right\} \\
\mathbf{n}_{1}=\operatorname{lin}\left\{E_{k l}+E_{l k}: l \leq k\right\}=M
\end{gathered}
$$

Then

$$
\mathbf{s}=\mathbf{n}_{1} \oplus \mathbf{n}_{0} \oplus \mathbf{a}=\mathbf{n}_{1}+\mathbf{s}_{0}
$$

is a Lie algebra, the Lie bracket being defined as follows:

$$
\begin{gathered}
{[E, F]=E F-F E \text { for } E, F \in \mathbf{s}_{0}} \\
{[X, Y]=0 \text { for } X, Y \in \mathbf{n}_{1}} \\
{[E, X]=E^{t} X+X E \text { for } E \in \mathbf{s}_{0}, X \in \mathbf{n}_{1} .}
\end{gathered}
$$

We see that the group

$$
S=\exp \mathbf{s}=N_{1} N_{0} A=N_{1} S_{0}
$$

acts on $D$ simply transitively :

$g(Y+i H)=g^{t} Y g+i g^{t} H g$ for $g \in S_{0}$ and $Y_{0}(Y+i H)=Y_{0}+Y+i H$ for $Y_{0} \in N_{1}$.

We notice that $N=N_{1} N_{0}$ is a nilpotent group on which $A$ acts diagonally, $\mathbf{n}_{0}$ and $\mathbf{n}_{1}$ are homogeneous subalgebras, and the basis $\left\{E_{i j}: i<j\right\} \cup\left\{E_{k l}+E_{l k}\right.$ : $k \leq l\}$ defines roots on $\mathbf{a}$.

ExAmple 4 . Let $\mathcal{C}_{2}$ be the space of complex $s \times t$ matrices, $\mathcal{C}_{1}$ - of complex $s \times s$ matrices. We have

$$
\mathcal{C}_{1}=\mathcal{H}+i \mathcal{H}
$$

where $\mathcal{H}$ is the real space of Hermitian $s \times s$ matrices and for a matrix $E \in \mathcal{C}_{1}$ we write

$$
E=\Re E+i \Im E,
$$

where $\Re E, \Im E \in \mathcal{H}$.

Let $\Omega$ be the open cone in $\mathcal{H}$ consisting of positive definite matrices, the nonnegative definite matrices being its closure.

If we define a Hermitian bilinear form

$$
\Phi: \mathcal{C}_{2} \times \mathcal{C}_{2} \ni E, F \rightarrow E F^{\star} \in \mathcal{C}_{1},
$$

then we see that $\Phi(E, E) \in \bar{\Omega}$ for all $E \in \mathcal{C}_{2}$ and $\Phi(E, E)=0$ implies $E=0$.

Let

$$
\begin{aligned}
& D=\left\{(E, F) \in \mathcal{C}_{1} \times \mathcal{C}_{2}: \Im E-\Phi(F, F) \in \Omega\right\}, \\
& B=\left\{(E, F) \in \mathcal{C}_{1} \times \mathcal{C}_{2}: \Im E-\Phi(F, F)=0\right\} .
\end{aligned}
$$

Let $A$ be the group of $s \times s$ diagonal matrices with non-zero entries, $N_{0}$ the group of unipotent upper triangular $s \times s$ matrices, $S_{0}=N_{0} A$. For $g \in S_{0}$ we define the action on $D$ by

$$
g(E, F)=\left(g^{*} E g, g F\right)
$$

It is clear that

$$
\Phi\left(g F_{1}, g F_{2}\right)=g \cdot \Phi\left(F_{1}, F_{2}\right) .
$$


The group $N_{1}=\mathcal{H} \times \mathcal{C}_{2}$ is defined as $N(\Phi)$ below.

The group $N_{1} N_{0} A$ acts simply transitively on $D$.

Example 4 shows all the main features of the so called homogeneous Siegel domains. The importance of the homogeneous Siegel domains is stressed by the fact, proved by Piateckij-Shapiro in $1960[\mathrm{P}]$, that these are precisely all the domains which are biholomorphic with the bounded homogenous domains.

As we see in Examples 3 and 4 above, our boundary $B$ of $D$ is much smaller than the topological boundary of $D$ as embedded in $\mathbf{C}^{n}$. However, it has the property that for every holomorphic bounded function $F$ on $D$ which extends continuously to $B$ we have

$$
\sup \{|F(z)|: z \in D\}=\sup \{|F(x)|: x \in B\} .
$$

Abusing slightly, the terminology we call $B$ the Shilov boundary of $D$ while in fact it is only a dense subset of the compact Shilov boundary in the bounded realization of $D$.

A Siegel domain (not necessarily homogeneous) is defined as follows:

Let $\Omega \subset \mathbf{R}^{n_{1}}$ be a regular cone, i.e. a nonempty open convex cone $\Omega$ with vertex at 0 and containing no entire straight line.

Given a regular cone in $\Omega$ in $\mathbf{R}^{n_{1}}$, we say that a Hermitian bilinear map

$$
\Phi: \mathbf{C}^{n_{2}} \times \mathbf{C}^{n_{2}} \rightarrow \mathbf{C}^{n_{1}}
$$

is $\Omega$-positive if $\Phi\left(z_{2}, z_{2}\right) \in \bar{\Omega}$ for all $z_{2} \in \mathbf{C}^{n_{2}}$ and $\Phi\left(z_{2}, z_{2}\right)=0$ implies $z_{2}=0$. The domain

$$
D=\left\{\left(z_{1}, z_{2}\right) \in \mathbf{C}^{n_{1}} \times \mathbf{C}^{n_{2}}: \Im z_{1}-\Phi\left(z_{2}, z_{2}\right) \in \Omega\right\}
$$

is called a generalized half-plane or a Siegel domain determined by $\Phi, \Omega$. This definition includes also the case where $n_{2}=0$. Then $D$ is a tube domain over $\Omega$, as in Examples 1 and 3.

What we call the Shilov boundary of $D$ is the set

$$
B=\left\{\left(z_{1}, z_{2}\right) \in \mathbf{C}^{n_{1}} \times \mathbf{C}^{n_{2}}: \Im z_{1}-\Phi\left(z_{2}, z_{2}\right)=0\right\} .
$$

As in Example 2, the group

$$
N(\Phi)=\mathbf{R}^{n_{1}} \times \mathbf{C}^{n_{2}}
$$

with multiplication

$$
\left(h_{1}, w_{1}\right)\left(h_{2}, w_{2}\right)=\left(h_{1}+h_{2}+2 \Im \Phi\left(w_{1}, w_{2}\right), w_{1}+w_{2}\right)
$$

acts on $D$ :

$$
(x, u)\left(z_{1}, z_{2}\right)=\left(z_{1}+x+2 i \Phi\left(z_{2}, u\right)+i \Phi(u, u), z_{2}+u\right) .
$$

Therefore $D=\{(x, u)(i t, 0):(x, u) \in N(\Phi), t \in \Omega\}$ and the decomposition $z=(x, u)(i t, 0)$ of a point $z \in D$ is unique. The orbits of $N(\Phi)$ are parametrized by the elements it, $t \in \Omega . N(\Phi)$ acts also simply transitively on $B$.

The Hardy spaces $H^{p}(D)$ are defined as follows. 
$H^{p}(D)$ is the set of all holomorphic $F: D \rightarrow \mathbf{C}$ such that

$$
\|F\|_{H^{p}(D)}^{p}=\sup _{t \in \Omega} \int_{N(\Phi)}|F((x, z)(i t, 0))|^{p} d x d z<\infty .
$$

Let

$$
F((x, z)(i t, 0))=F_{t}(x, z) .
$$

Since for $F \in H^{p}(D)$ the norms $\left\|F_{t}\right\|_{L^{p}(N(\Phi))}$ are bounded, for $p>1$ there exists a function $F_{0}$ such that

$$
\lim _{t \rightarrow 0}\left\|F_{t}-F_{0}\right\|_{L^{p}(N(\Phi))}=0 .
$$

It follows that the map $F \rightarrow F_{0}$ is one-to-one from $H^{2}(D)$ onto a closed subspace of $L^{2}(F)$. Thus $H^{2}(D)$ is a Hilbert space, the inner product being defined by

$$
\langle F, G\rangle=\lim _{t \rightarrow 0} \int_{F} F_{t}(\xi) \bar{G}_{t}(\xi) d \xi=\left\langle F_{0}, G_{0}\right\rangle_{L^{2}(B)} .
$$

$H^{2}(D)$ has a reproducing kernel $S(z, w)$ defined on $D \times D$ called the Szegö kernel $[\mathrm{H}] . S(z, w)$ is holomorphic with respect to $z=\left(z_{1}, z_{2}\right)$ and for fixed $w$, $S(\cdot, w) \in H^{2}(D)$. A formula for $S(z, w)$ proved in [KS2] gives $S(z, w)$ in terms of a "Fourier transform" over the dual cone $\Omega^{*}$.

$$
S^{w}(z)=S(z, w)=\int_{\Omega^{*}} e^{-2 \pi\langle\lambda, \rho(z, w)\rangle} \operatorname{det} \Phi_{\lambda}^{\frac{1}{2}} d \lambda,
$$

where

$$
\rho(z, w)=i\left(\bar{w}_{1}-z_{1}\right)-2 \Phi\left(z_{2}, w_{2}\right)
$$

If $n_{2}=0$ then

$$
S^{w}(z)=\int_{\Omega^{*}} e^{-2 \pi\langle\lambda, \rho(z, w)\rangle} d \lambda
$$

For given $w$, let $S_{0}^{w}$ be the limit of $S_{t}^{w}$ in $L^{2}(B)$. Then $S^{w}(x)=S_{0}(x, w)$ and $S_{0}$ is a function on $D \times B$, holomorphic with respect to $z$ and square integrable with respect to $x$, such that for every $F$ in $H^{2}(D)$

$$
F(z)=\int_{B} S_{0}(z, x) f(x) d x
$$

for some $f \in L^{2}(B)$. The Poisson kernel for $D$, introduced by Hua, is the function $P(x, z)$ on $B \times D$ defined by

$$
P(x, z)=\frac{\left|S_{0}(x, z)\right|^{2}}{S(z, z)}, \quad x \in B, z \in D .
$$

For every $z$

$$
\int_{B} P(x, z) d x=1 .
$$


Every $F \in H^{p}, 1 \leq p \leq \infty$, is a Hua-Poisson integral of a function $f \in L^{p}$, i.e.

$$
F(z)=\int_{B} f(x) P(x, z) d x .
$$

Also for every $f \in L^{p}(B)$ the function $F$ above is a function on $D$. It is called the Hua-Poisson integral of $F$.

We see that the definitions given above are modeled on the case of one complex variable (Example 1). We have

$$
\begin{gathered}
S(z, x)=\frac{1}{2 \pi i} \frac{1}{x-z}=\frac{1}{2 \pi i} \frac{1}{x-y-i a}, \text { if } z=y+i a \\
P(z, x)=\frac{1}{\pi} \frac{a}{(y-x)^{2}+a^{2}},
\end{gathered}
$$

in which we recognize the Cauchy kernel and the ordinary Poisson kernel.

We also note that both are convolution kernels on the group $N(\Phi)$ and rewrite the formula above as

$$
\int_{N} f(x) P(y \cdot t, x) d x=\int_{N} f\left(y^{-1} x\right) P_{t}(y) d y=f \star P_{t}(x), \quad t \in \Omega .
$$

This is true in general, the Hua-Poisson kernel can be rewritten as a family of convolution kernels $P_{t}, t \in \Omega$ on $N(\Phi)$.

Except for the very classical cases, there are no explicite formulas neither for the Szegö nor for the Hua-Poisson kernel. However much is known about both of them. The main tool here is formula (1.2) for the Szegö kernel.

Here are some estimates of the Hua-Poisson kernels which we were able to obtain for general Siegel domains, [DHP1].

Let $\|\cdot\|$ be a norm in $N(\Phi)$. For a fixed $t \in \Omega$ we have

$$
\text { There exists } \eta>0 \text { such that } \int_{N(\Phi)}\|y\|^{\eta} P_{t}(y) d y<\infty .
$$

For every multiindex $I$ there are constants $c, M$ such that

$$
\left|\partial^{I} P_{t}(y)\right| \leq c(1+\|y\|)^{M} .
$$

There exist $c, \epsilon>0$ such that $P_{t}(y) \leq c(1+\|y\|)^{-\epsilon}$.

Properties (1.3) - (1.5) are the only pointwise estimates for $P$ which we can prove in the case of the general Siegel domains. However we have proved that they are sufficient to obtain a satisfactory Fatou type theorem about almost everywhere convergence of the Hua-Poisson integrals of functions in $L^{p}, 1<p \leq \infty$, to their boundary values, [DHP1].

Another thing which is of importance here is that due to a version of the Harnack inequality for the Hua-Poisson integrals, a consequence of the mean 
value theorem for holomorphic functions, the maximal function

$$
M f(x)=\sup _{t \in A K} P_{t} \star f(x), \text { where } K \text { is a compact subset in } \Omega
$$

is dominated by

$$
M^{\prime} f(x)=\sup _{t \in \Lambda K} P_{y} \star f(x),
$$

where $\Lambda=\left\{\left(e^{m_{1}} \mathbf{e}_{1}, \ldots, e^{m_{n}} \mathbf{e}_{n}\right): m_{j} \in \mathbf{Z}\right\}, \mathbf{e}_{1}, \ldots, \mathbf{e}_{n}$ form an appropriate basis in the vector group $A$.

Theorem 1 (E. Damek, A. Hulanicki, R. Penney [DHP1]). For $f \in L^{p}(B)$, $1<p \leq \infty$, the Hua-Poisson integral

$$
\int P(x \cdot(i t, 0), y) f(y) d y \rightarrow f(x) \text { almost everywhere }
$$

as $t \rightarrow 0$ in an appropriate way inside the cone $\Omega$.

The Hua-Poisson integrals are functions on $D$ which are called Hua-harmonic, Hua-harmonic functions are annihilated by the Laplace-Beltrami operator $\Delta$ (with respect to the Bergman metric on $D$ ) in the case of the symmetric domains. However, the class of $\Delta$-harmonic functions on $D$ is much larger, in general. In 1975 A. Korányi and P. Malliavin $[\mathrm{KM}]$ presented two elliptic operators $L_{1}$ and $L_{2}$ on the Siegel domain of $2 \times 2$ symmetric matrices as in Example $2, n=2$, such that the set of bounded functions annihilated by both $L_{1}$ and $L_{2}$ are the Hua-Poisson integrals of $L^{\infty}$ functions on $B$. In 1980 K. Johnson and A. Korányi $[\mathrm{JK}]$ found for symmetric tube domains an elliptic system whose zeros are precisely the Hua-harmonic functions.

Theorem 2 (E. Damek, A. Hulanicki, R. Penney [DHP2]). For the domain of our Example 2 (general $n \times n$ matrices) there exists an elliptic real second order differential operator $L$ on $D$ such that bounded Hua-harmonic functions are precisely the bounded functions $F$ such that $L F=0$.

2. Harmonic functions. Let $L$ be a second order left-invariant degenerate elliptic operator without a constant term on $S$ :

$$
L=X_{1}^{2}+\ldots+X_{m}^{2}+X_{0} .
$$

We shall assume that $X_{0}, X_{1}, \ldots, X_{m}$ satisfy the Hörmander condition, i.e. the smallest Lie subalgebra which contains $X_{0}, \ldots, X_{m}$ is equal to $\mathbf{s}$. We write

$$
X_{0}=Y_{0}+Z_{0}, \quad Y_{0} \in \mathbf{n}, Z_{0} \in \mathbf{a} .
$$

Now let

$$
\Lambda_{0}=\left\{\lambda \in \Lambda:\left\langle\lambda, Z_{0}\right\rangle \geq 0\right\}
$$

We define a subalgebra

$$
\mathbf{n}_{0}(L)=\oplus_{\lambda \in \Lambda_{0}} \mathbf{n}^{\lambda}
$$


and the corresponding subgroup

$$
N_{0}(L)=\exp \mathbf{n}_{0}(L) .
$$

Let $\mathbf{n}_{0}$ be an arbitrary homogeneous subalgebra of $\mathbf{n}$ containing $\mathbf{n}_{0}(L)$ and let

$$
N_{0}=\exp \mathbf{n}_{0} .
$$

Let $\left\{\mu_{t}\right\}_{t>0}$ be a semigroup of probability measures generated by $L$. Let $\left\{S_{t}(\omega)\right\}$ be the diffusion process defined by the semigroup $\left\{\mu_{t}\right\}_{t>0}$ on $S$.

Definition of the Furstenberg BOUndary. Let $X$ be a locally compact space with a probability measure $\sigma$. Assume that $S$ acts transitively on $X$. $X$ is called a boundary for the pair $S, L$ if

$$
\text { for every } t>0 \quad \breve{\mu}_{t} \star \sigma=\sigma
$$

and

$$
\lim _{t \rightarrow \infty} \int f\left(S_{t}(\omega) x\right) d \sigma(x)=f(x(\omega))
$$

for almost every trajectory $S_{t}(\omega)$, where $x(\omega)$ is a point of $X$ depending only on $\omega$. A Poisson boundary for $L$ is the maximal Furstenberg boundary $X$. by

Formula (2.2) implies that for every $f \in L^{\infty}(\sigma)$ the function $F$ on $S$ defined

$$
F(s)=\int_{X} f(s x) d \sigma(x)
$$

is $L$-harmonic.

If $X$ is the Poisson boundary, then every bounded harmonic function $F$ on $S$ is of the form (2.3).

In [DH1] we have shown that the boundaries of the pair $S, L$ are precisely the $S$-spaces

$$
X=S / N_{0} A=N / N_{0} .
$$

Let us elaborate this fact. We write $S \times X \ni(s, u) \mapsto s u \in X$ for the natural action of $S$ on the quotient space $X$. We select a point $e$ in $X$ and define the map

$$
\mathbf{p}: S \ni s \mapsto s e \in X .
$$

For a measure $\nu$ on $X$ and a bounded measure or a distribution with compact support $\mu$ on $S$ we write $\mu * \nu$ for the natural convolution corresponding to this action. We have, cf. [DH1]:

$$
\sigma \text { is a }{ }^{*} \text {-weak limit of } \mathbf{p}\left(\breve{\mu}_{t}\right) \text { as } t \rightarrow \infty \text {. }
$$

We see that in our case $X=\mathbf{R}^{\chi}$ as a manifold. Moreover,

$$
\sigma \text { has a smooth density } d \sigma(x)=P(x) d x,
$$


where $d x$ is the Lebesgue measure. Let $f$ be a function on $X$ and suppose $f \in$ $L^{p}\left(\mathbf{R}^{\chi}\right)$ for some $p, 1 \leq p \leq \infty$. Then (2.3) becomes

$$
F(s)=\int_{X} f(s \cdot x) P(x) d x,
$$

we call it the Poisson integral of $f$ and

$$
P(s, x)=\frac{d s^{-1} \cdot x}{d x} P\left(s^{-1} \cdot x\right)
$$

the Poisson kernel.

In our Example 3 the domain $D$ is identified with $N A$ where $N=N_{0} N_{1}$, $N_{0} \subset \Omega$ consists of matrices with ones on the diagonal and $A$ are the diagonal matrices in $\Omega$.

For the proof of Theorem 1 we exhibit an operator of the form (2.1) such that the roots defined by the homogenous basis of $M$ are negative on $Z_{0}$, cf. (2.2), and the ones defined by the homogeneous basis of $\mathbf{n}_{0}$ are non-negative. Of course the operator has also to annihilate Hua-harmonic functions on $D$.

3. Estimates for the Poisson kernel. The examples discussed above point to the importance of the Poisson kernels on the Furstenberg boundaries which in the case of non-symmetric Siegel domains do not coincide with the Hua-Poisson kernel (i.e. normalized square of the modulus of the Szegö kernel).

The first thing we can tell about them for general $N A$ groups is that they satisfy estimates (1.3)-(1.5).

Properties (1.3) - (1.5) are the only estimates for $P$ we can prove in the case of general $N A$ groups. However, we have proved that they suffices to prove a satisfactory Fatou type theorem on almost everywhere convergence of the Poisson integrals of functions in $L^{p}, 1<p \leq \infty$, to their boundary values.

We note that in general a boundary is only a homogeneous space $N / N_{0}$, not necessarily a subgroup of $N$ which is the case for the Poisson boundary. This is the reason why in the general case the proof of the Fatou theorem is much more complicated, cf. [DH3]. In fact, to estimate the maximal functions involved we have to consider maximal functions along surfaces. Here the ideas and techniques of M. Christ [Ch1], [Ch2], are extremely useful.

On the other hand, for the case when $N A$ is a harmonic space and $L$ is the Laplace-Beltrami operator, E. Damek and F. Ricci have proved a formula for $P$ very similar to the corresponding one for symmetric spaces of rank one.

Their result is

$$
P(x, z)=c \frac{1}{\left.\left(1+\frac{1}{4}|x|^{2}\right)^{2}+|z|^{2}\right)^{Q}} \simeq c\|x, z\|^{-2 Q},
$$

where $|\cdot|$ is a specific homogenous gauge on $N$ and $Q$ is the homogenous dimension of $N$. 
Formula (3.1) has been a starting point for a search of better estimates on $P$ and their derivatives first in the case of one-dimensional $A$.

In this case we identify $A$ with $\mathbf{R}^{+}, N$ becomes a homogeneous group on which $A$ acts by dilations, i.e. there is a

$$
\text { homogeneous basis } Y_{1}, \ldots, Y_{n} \text { of } \mathbf{n}
$$

and positive numbers $1=d_{1} \leq \cdots \leq d_{n}$ such that

$$
\delta_{a}\left(\exp \sum_{j=1}^{n} y_{j} Y_{j}\right)=\exp \left(\sum_{j=1}^{n} y_{j} a^{d_{j}} Y_{j}\right), \quad a \in A
$$

are automorphisms of $N$. Let $Q=d_{1}+\ldots+d_{n}$ be the homogeneous dimension of $N$.

In this case operator $(2.1)$ can be written in the form

$$
L f(x a)=\left(\left(a \partial_{a}\right)^{2}-\alpha a \partial_{a}+\sum_{i, j=1}^{n} \alpha_{i, j} a^{d_{i}+d_{j}} Y_{i} Y_{j}+\sum_{i=1}^{n} \alpha_{i} a^{d_{i}} Y_{i}\right) f(x a),
$$

where the matrix $\left[\alpha_{i j}\right]$ is non-negative definite.

If $L$ is the Laplace-Beltrami operator on $N A$ then $\alpha=Q$.

Recently, using the method by which Alano Ancona $[\mathrm{A}]$ described the minimal positive harmonic functions on Riemannian spaces of negative curvature, E. Damek has proved the following

TheOREM 3 (E. Damek [D2]). If an operator $L$ is of the form (3.2), then

$$
c^{-1}(1+|x|)^{-\alpha-Q} \leq P(x) \leq c(1+|x|)^{-\alpha-Q}
$$

for some constant $c$.

The proof of the theorem is based on a boundary Harnack inequality for positive harmonic functions on $N A$ and cannot be used to derive estimates for the derivatives. Therefore Ewa Damek, Andrzej Hulanicki and Jacek Zienkiewicz revised a probabilistic approach to the Poisson kernel used earlier by E. Damek and A. Hulanicki [DH2].

Let

$$
\left.L_{t}=\sum_{i, j=1}^{n} \alpha_{i, j} a(t)^{d_{i}+d_{j}} Y_{i} Y_{j}+\sum_{i=1}^{n} \alpha_{i} a(t)^{d_{i}} Y_{i}\right)
$$

where $a(t)$ is a trajectory with $a(0)=1$ of the Brownian motion on $\mathbf{R}^{+}$generated by

$$
\left(a \partial_{a}\right)^{2}-\alpha a \partial_{a}
$$

Then the following holds: 
Let $p(\cdot ; s, t), s<t$, be a non-negative function on $N$ such that for every $f \in C_{c}^{\infty}(N)$ the function $u(x ; s, t)=f * p(x ; s, t)$ solves

$$
L_{t} u(x ; s, t)=\partial_{t} u(x ; s, t), \lim _{t \nearrow s} u(x ; s, t)=f(x) .
$$

Of course $p(\cdot ; s, t)$ depends on the trajectory $a(\cdot)$. Then taking the expected value with respect to the Wiener measure we obtain

$$
\operatorname{Ep}(x ; 0, \infty)=P(x) .
$$

We have been able to obtain good estimates for the kernels $p(\cdot ; 0, \infty)$ in terms of the numbers

$$
A_{j}=\int_{0}^{\infty} a(t)^{d_{j}} d t
$$

and the explicit formula for the distribution of the Brownian random variables $A_{j}$. By this method we have proved the upper bound of (3.4) and

Theorem 4 (E. Damek, A. Hulanicki, J. Zienkiewicz). For every multiindex I there is a constant $C_{I}$ such that

$$
\left|Y^{I} P(x)\right| \leq C_{I}(1+|x|)^{-\alpha-Q-|I|} .
$$

The problem of finding precise pointwise estimates for the Poisson kernel in the case when $A$ is multidimensional is still wide open, cf. also [St].

\section{References}

[A] A. Ancona, Negatively curved manifolds, elliptic operators, and the Martin boundary, Ann. of Math. (2) 125 (1987), 495-536.

[Ch1] M. Christ, Hilbert transforms along curves I. Nilpotent groups, Ann. of Math. (2) 122 (1985), 575-596.

[Ch2] M. Christ, The strong maximal function on a nilpotent group, Trans. Amer. Math. Soc. 331(1) (1992), 1-13.

[CW] R. R. Coifman and G. Weiss, Operators associated with representations of amenable groups, singular integrals induced by ergodic flows, the rotations method and multipliers, Studia Math. 47 (1973), 285-303.

[D1] E. Damek, Left-invariant degenerate elliptic operators on semidirect extensions of homogeneous groups, Studia Math. 89 (1988), 169-196.

[D2] E. Damek, Pointwise estimates on the Poisson kernel on NA groups by the Ancona method, to appear.

[DH1] E. Damek and A. Hulanicki, Boundaries for left-invariant subelliptic operators on semi-direct products of nilpotent and Abelian groups, J. Reine Angew. Math. 411 (1990), 1-38.

[DH2] E. Damek and A. Hulanicki, Maximal functions related to subelliptic operators invariant under an action of a solvable Lie group, Studia Math. 101 (1991), 33-68.

[DH3] E. Damek and A. Hulanicki, Boundaries and the Fatou theorem for subelliptic second order operators on solvable Lie groups, Colloq. Math. 68 (1995), 121-140.

[DHP1] E. Damek, A. Hulanicki, R. Penney, Admissible convergence for the PoissonSzegö integrals, J. Geom. Anal. (to appear) 
[DHP2] E. Damek, A. Hulanicki, R. Penney, Hua operators on bounded homogeneous domains in $\mathbf{C}^{n}$, preprint.

[DR1] E. Damek and F. Ricci, A class of nonsymmetric harmonic Riemannian spaces, Bull. Amer. Math. Soc. 27 (1992), 139-142.

[DR2] E. Damek and F. Ricci, Harmonic analysis on solvable extensions of $H$-type groups, J. Geom. Anal. 2 (1992), 213-248.

[H] L. K. Hua, Harmonic Analysis of Functions of Several Complex Variables in Classical Domains, Vol. 6, Translations of Math. Monographs, Amer. Math. Soc., Providence, 1963.

[JK] K. Johnson, A. Korányi, The Hua operators on bounded symmetric domains of tube type, Ann. of Math. (2) 111 (1980), 589-608.

[K1] A. Korányi, The Poisson integral for generalized halfplanes and bounded symmetric domains, Ann. of Math. (2) 82 (1965), 332-350.

[K2] A. Korányi, Boundary behavior of Poisson integrals on symmetric spaces, Trans. Amer. Math. Soc. 140 (1969), 393-409.

[K3] A. Korányi, Harmonic functions on symmetric spaces, in: Symmetric Spaces, BaselNew York 1972.

[KM] A. Korányi and P. Malliavin, Poisson formula and compound diffusion associated to overdetermined elliptic system on the Siegel half-plane of rank two, Acta Math. 134 (1975), 185-209.

[KS1] A. Korányi, E. M. Stein, Fatou's theorem for generalized half-planes, Ann. Scuola Norm. Sup. Pisa 22 (1968), 107-112.

[KS2] A. Korányi, E. M. Stein, $H^{2}$-spaces of generalized half-planes, Studia Math. 44 (1972), 379-388.

[NS] A. Nagel and E. M. Stein, On certain maximal functions and approach regions, Adv. Math. 54 (1984), 83-106.

[P] I. I. Pjatecki-Shapiro, Geometry and classification of homogeneous bounded domains in $\mathbf{C}^{n}$, Uspekhi Mat. Nauk 2 (1965), 3-51; Russian Math. Surv. 20 (1966), $1-48$.

[R] F. Ricci, Singular integrals on $\mathbf{R}^{n}$, Tempus lectures held at the Institute of Mathematics of Wrocław University, 1991.

[Sj] P. Sjögren, Admissible convergence of Poisson integrals in symmetric spaces, Ann. of Math. (2) 124 (1986), 313-335.

[S] J. Sołowiej, The Fatou theorem for NA groups - a negative result, Colloq. Math. 67 (1994), 131-145.

[St] E. M. Stein, Boundary behavior of harmonic functions on symmetric spaces: Maximal estimates for Poisson integrals, Invent. Math. 74 (1983), 63-83.

[V] E. B. Vinberg, The theory of convex homogeneous cones, English translation, Trans. Moscow Math. Soc. 12 (1963), 340-403. 